\title{
VIEWPOINT
}

\section{Should we consider non-compliance a medical error?}

\section{N Barber}

Qual Saf Health Care 2002;11:81-84

Non-compliance is an extensive intractable problem. This paper argues that we can gain significant insight into non-compliance if we apply theories developed to explain human error in organisations. The resultant framework encompasses intentional and unintentional non-compliance, shifts blame from the patient, and recognises the influence of other factors, including organisational ones. There are also consequences for the measurement of compliance and new strategies to improve it. Terminology will need to be addressed, particularly whether intentional non-compliance by a patient should be considered an error. If empirical research supports the arguments in this paper then, with some further theory development, the application of human error theory will offer a useful new approach to understanding and reducing undesired non-compliance.

Correspondence to:

Professor N Barber, Centre for Practice and Policy, The School of Pharmacy,

29-39 Brunswick Square, London WCIN IAX, UK nick.barber@ulsop.ac.uk

Accepted for publication 10 January 2002
A round one third to one half of patients do not take their medicines as directed, yet this is not usually considered to be a medical error. Should this be the case? This article explores whether it should, and argues that there are benefits in applying the literature on errors to non-compliance.

Non-compliance is a substantial and, as yet, intractable issue; any new insights into it are to be welcomed. Estimates have remained constant over the years, that $30-50 \%$ of patients on chronic medication do not take their medicines as directed. ${ }^{12}$ We do not know the full consequences of non-compliance on the population as a whole. The economic studies have been limited, but all of them suggest substantial consequences. In 1985 Smith estimated cardiovascular non-compliance alone resulted in 125000 deaths in the USA annually and a further \$1.5bn in lost earnings from hospitalisations. ${ }^{3}$ Another study suggested non-compliance to just 10 drugs cost the USA between \$396 and \$792 million each year, ${ }^{4}$ and the overall cost to the USA of all non-compliance is estimated at \$100bn annually. ${ }^{5}$

The mutual exclusion of non-compliance and errors is fairly comprehensive. I failed to find any mention of non-compliance in a quick skim of my books on error, so I searched Medline over the last quarter century. Using the terms "patient compliance OR medication errors" there were 24702 references; searching for "patient compliance AND medication errors" and restricting the search to "English" and "journal article" reduced the number to just 50. Most of these were studies in which the authors simply regarded noncompliance as an error but did not address it with reference to any literature on errors. An exception was the American Society of Hospital Pharmacists which did include non-compliance in its guidelines on preventing medication errors in hospitals. ${ }^{6}$ Considering the development of the literature on the causes of errors, the growing application of human error theory in medicine, and national initiatives to reduce errors in the UK and USA, it seems a good time to reassess whether the overlap should be so small.

\section{NON-COMPLIANCE}

To understand the reasons for the separation of these two literatures, we need first to understand the definitions and causes of non-compliance. Compliance is usually defined as the extent to which patients follow medical advice. ${ }^{7}$ While often used with respect to medicine taking (as I will use it in this article), it need not be so, and could apply to the following of any advice on health. This commonly used definition is based on patient behaviour (an outcome based definition also exists ${ }^{8}$ ), and is rather like saying someone has a "stomach ache" -while it expresses a condition, it is not clear how serious it is and there may be many causes, each of which may require different solutions. Non-compliance to medication is often set at some level-for example, that less than $80 \%$ of doses are taken correctly. While this definition is precise, if somewhat arbitrary, it is difficult to assess as it is hard to find the true nature of patients' behaviour.

The term "compliance" is itself controversial, and this controversy is informative. In the 1970s it was felt that doctors told patients the right thing to do, and that patients should comply with these directions. However, Stimson, ${ }^{9}$ in a seminal paper, argued that it was inappropriate to see patients as "defaulters" and he put forward the patient's side of the argument. More recently the term "adherence" has been suggested as being more suitable than compliance, as it is seen to lack the implied one sided value implications of "compliance". Adherence became more popular, particularly with researchers, but it was never that familiar to practitioners and so has been limited in its use. More recently, with the growth of interest in patients participating in decision making, the objections to "compliance" are weakened (as it is compliance with, ideally, an agreed regime), and the term seems to be becoming popular again. "Concordance" is another term which is sometimes used, inappropriately, as a synonym for compliance. In a document entitled "From compliance to concordance"10 a specific form of doctorpatient interaction about medicines was suggested, and it was inferred that this would improve compliance. Perhaps because of the title of the article, some people incorrectly assume 


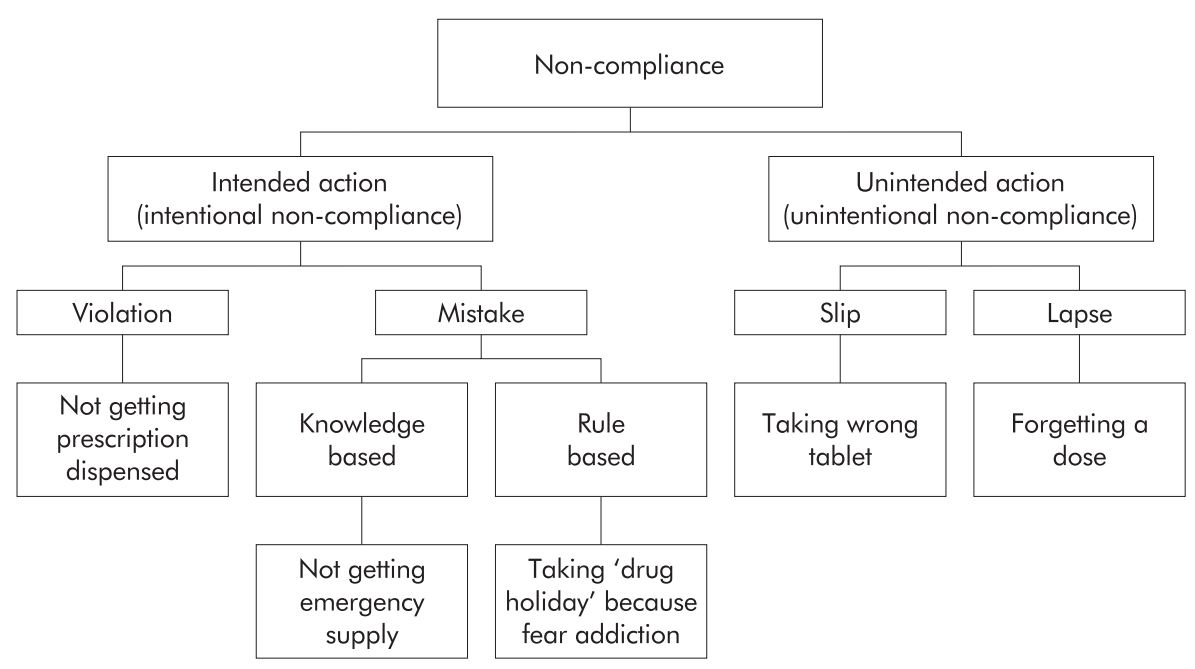

Figure 1 Non-compliance illustrated using Reason's framework.

concordance is a modern word for compliance. These definitional arguments are not just academic semantics, but have at their core the role of the patient's free will, and whether the exercising of free will can be considered an error. This issue will be discussed later in this paper.

About 200 factors have been attributed to cause noncompliance. ${ }^{11}$ However, the causes-and hence the patientsare usually split into two groups: intentional and unintentional non-compliers. Those who intentionally do not comply have made the decision consciously; the others wish to comply but cannot do so for some reason. In reality there is some overlap between these groups. For example, some patients who forget to take their medicine (which would be classed as unintentional non-compliance) will do so because their view of the medicine, its importance and risks, may have made taking the medicine a low priority, and hence more easily driven out of mind by other matters.

What do we know of patients' intentions? The trend in recent times has been for psychologists to focus on the causes of intentional, rather than unintentional, non-compliance. They have applied several theoretical models to explain non-compliance ${ }^{12}$-for example, social cognition models, stage models, and Leventhal's self-regulatory theory. These do have several limitations and are not particularly good at predicting actual behaviour. When models allow regression modelling, in which the extent of compliance is the outcome variable, they generally account for a relatively small proportion of the observed variability. One reason for their limited success may be that they apply a theory to all non-compliers, whether intentional or unintentional. We would expect these models to predict intentional noncompliance much more than unintentional. Unless a substantial proportion were intentionally non-compliant, we would expect the noise from the others to weaken the explanatory power of the model. Although the division of non-compliance into intentional and unintentional is of long standing, I have been unable to find any papers that report the proportion of non-compliance in each group, so we still do not know how well we would expect the models to work. This is obviously of importance if we wish to reduce non-compliance. Different causes will require different solutions. While these theoretical approaches have helped our understanding of compliance, as Horne and Weinman note, ${ }^{12}$ the capacities of the models to generate effective interventions have yet to be fully evaluated.

The intractability of non-compliance is another clue that a new approach may be welcome. A Cochrane review ${ }^{13}$ found that there had been few well conducted studies, and that many interventions failed. Only one study of short term treatment was effective and, of the 17 randomised controlled trials of long term treatment that were of sufficient quality, only nine led to improvements in treatment outcome. Nearly all the successful studies used complex mixtures of interventions.

The current explanatory models of non-compliance are limited and there is a lack of effective solutions; there is room for a new approach. Having conducted research into non-compliance and, separately, into human factors in medical error, I find the stories of non-compliant patients remind me of the stories of those who have made errors. So far I have been unable to find any application of human error theory to non-compliance. In the next sections I describe current thinking on the causes of errors, illustrate how these explanatory models and techniques could explain non-compliance, suggest how the approaches could inform research, and outline some difficulties with the approach.

\section{MEDICAL ERROR THEORY}

This analysis is based on Reason's descriptions of the causes of human error ${ }^{14}{ }^{15}$ which are adapted to non-compliance in fig 1. In Reason's terms, unsafe human acts follow intended or unintended actions (equivalent to intentional or unintentional non-compliance). Unintentional actions are made up of slips and lapses. These occur when people intend to do an action but do not do it properly; they occur largely in the automatic carrying out of routine tasks. Slips are a result of a lack of attention-for example, inadvertently taking the wrong tablet. Lapses result from failure of memory such as forgetting to take a dose.

Mistakes are intended actions that are wrong, but are carried out with the intention of doing the right thing, so the perpetrator is unaware that what they are doing is wrong; these would be classified as intentional non-compliance. They occur once a problem has been recognised in the current plan. There are two types of mistake-rule based and knowledge based. Rule based mistakes, by far the more common, occur when the person is operating to a set of rules, but the rule they use is wrong; knowledge based mistakes are rarer, and occur in novel situations when the person is faced with a problem and has to work out what to do from their existing knowledge, rather than knowing a rule that fits the situation. Rule based mistakes include misapplication of a good rule and application of a bad rule. An example from non-compliance would be that some patients on chronic medication become concerned about addiction ${ }^{16}$ and therefore deliberately stop taking a drug for a period each year (sometimes called a "drug holiday") even though the drug is not addictive. Application of a bad rule can occur in the case of inhaled steroids; I have met patients who, having heard about the effects of steroid abuse in sport, fear all steroids so choose not to take their medicine as frequently as directed. Knowledge based failures would include a patient 
who runs out of an important medicine (for some reason that is not their fault) and is faced with a decision of what to do; they may err by choosing to wait several days before seeing their doctor rather than getting an emergency supply from a pharmacy.

The final category of intentional acts/non-compliance is that of violations; these are deliberate deviations from safe practice. The boundary between violations and mistakes is rather blurred, but a guide is obtained by considering whether the error occurs solely from "internal processing", in which the person is trying to do the right thing but does not (a mistake), or whether the issue is more motivational and caused by their operating in a wider social context (a violation). A deliberate violation would be choosing not to get a prescription dispensed because the patient felt the doctor hadn't listened and so could not have understood the problem. Other violations include taking "short cuts" that bend the rules to make life easier, such as taking several medicines together which should be taken at different times, or taking a non-steroidal anti-inflammatory drug on an empty stomach. Violations need not be bad and, indeed, can avoid harm. Deliberate non-compliance can be the right thing for a patient to do, such as not taking a diuretic before a long bus journey.

Following the categorisation of errors and violations, Reason and others have gone on to consider the aetiology of accidents in an organisation. Organisational processes and management decisions create an environment that may encourage errors; these failures are known as latent errors as they create the background against which errors are more likely to occur. Partly as a result of the latent failures, there are also local conditions at the time of the accident which may increase the likelihood of a worker making an error. While much of this theory has been derived from studies of accidents in areas such as nuclear power, aviation and shipping, it also applies to health care. The patient, even though at home, has engaged with and is part of-indeed, the end point of-a large organisation with the goal of improving health. Human error theory is not the only body of learning to address these areas. Theories in psychology include attitude and "norms"; sociology includes organisational and social issues; however, error theories seem best to pull them all together.

The organisational model presents the people who make the error as "the inheritors rather than the instigators" of an accident. ${ }^{15}$ Consequently, in health care there is a widening from studying the person who made the error to also seeking organisational and local factors that caused them to make the error. There is the need for a similar shift in noncompliance-we should shift the focus from the patient to their local environment and the organisation of care supporting them. There are differences, of course. Contributing local factors in a hospital, such as the shift system, being short staffed that day, poor lighting etc, may have equivalents in the patient's home, work or life in general, but may well not be the responsibility of the healthcare system. However, this approach can direct our attention to the causes of noncompliance and to suggestions of ways in which the patient or others could resolve them.

Examples of latent failures could be the doctor's style of communication with the patient ${ }^{17}$ or the remuneration system for pharmacists, which leads to many of them dispensing large numbers of prescriptions at the cost of helping smaller numbers of patients become compliant (although there are suggestions which would change this in the current NHS plans for pharmacy ${ }^{18}$ ). Non-compliance resulting from the system of information transfer on discharge of patients from secondary to primary care is common. ${ }^{19}$ Local factors could be related to where the patient kept their medicines or how they decided to ask for repeat prescriptions (for example, the length of time before their medicines ran out that they asked for them), or the use of a carer to ensure the medicines were given.

\section{IMPLICATIONS FOR THEORY AND PRACTICE}

One advantage of the recognition that organisational factors can produce errors has been a shift away from blaming the individual who made the error. This is not only fairer (in the majority of cases), but also has the pragmatic benefit that it encourages people to be open about errors and, if linked to a monitoring mechanism, can lead to safer systems. A similar benefit could accrue if we adopted the same mechanism for non-compliant patients. I suspect little has changed since Stimson observed that non-compliant patients were seen as "deviants" by doctors. ' We need a mechanism to remove this stigma from patients and to encourage them to speak openly about how they use their medicines. We need to capture the information and learn from it.

This is reinforced by another area in which non-compliance research can benefit from the study of errors - that of deciding what we should measure and be concerned about. In studies of non-compliance the term is usually defined as a patient taking less than $80 \%$ of their doses as intended. This proportion presumably derives from the knowledge that most drugs follow a log dose response curve, so the reduction in clinical effect would only be likely to be evident when the total dose fell below about $80 \%$. This suggests the term "noncompliance" has been used to conflate two terms, one reflecting incidence and the other severity. As with all such measures, it provides a simple dichotomy but it raises the issue of whether this dichotomy is helpful. I would suggest that it is limited as it imposes a threshold before studying errors, and its link to outcome is tenuous. In contrast, it has been recognised in organisations that, while major errors are rare and therefore hard to study, they are often unusual concatenations of commoner minor errors. There is a direct relationship (often drawn as a pyramid) that, for every case of an error causing major harm (shown at the tip of the pyramid), there are proportionately larger and larger numbers of incidents producing less and less harm (shown in layers beneath the tip). One study showed that, for every case of major harm, there were seven injuries and 189 non-injury accidents. ${ }^{20}$ The number of harmful incidents can therefore be reduced be reducing the number of minor errors.

What can this teach us about non-compliance? Firstly, that all cases of non-compliance are of interest, whether one dose or many, so the use of the $80 \%$ cut off is unhelpful. Minor errors are easier to study and hence to understand their causes and devise remedial solutions; by reducing their incidence it is possible to reduce the incidence of major errors. This approach would seem likely to bear fruit in studying non-compliance. If all cases of missed and inappropriate doses are studied, we will be likely to identify common causes including intentional, unintentional, and latent organisational ones. No theoretical explanation of non-compliance has been able to do this so far. We can then devise better solutions that, by reducing the incidence of minor "errors", will also reduce the harm caused by non-compliance.

Removal of the $80 \%$ threshold has another advantage. It forces us to make explicit the relationship between noncompliance and harm. Instead of just counting the incidence, we should also be focusing on the effect it has on patient health. Why do some cases lead to hospitalisation or death and others do not? More empirical work is needed in this area.

One final implication of the influence of the organisational model on non-compliance is that it affects the design and interpretation of research. A study of several hundred patients of one doctor is less likely to be useful than a study of fewer patients from a greater number of doctors' practices.

While I have been emphasising similarities between the application of human error theory to errors in complex organisations and a patient taking their medicines, there are some significant differences. An organisation is trying to produce some end point in a safe manner, which it does by controlling the systems and activities of its staff. If someone 


\section{Key messages}

- Non-compliance is an extensive, intractable problem

- Methods of studying human error in organisations may be applicable to non-compliance.

- The consequences may be new insights into the causes of and solutions to non-compliance.

- This paper is based on an argument; theory development and empirical support are required next.

delivering health care commits an error, it is usually somebody else who suffers. A patient is different; they are both the actor in a process and the end point of that process, and if they make an error, it is usually they who suffer most. What is more, the end point of that process is defined by the patient, or at least influenced by them; it is the patient who first seeks treatment, initiating the process which leads to them being directed to take a medicine. If they choose to violate the dose directions, then in the majority of cases it is their right-they chose to start the process, so they can exercise their free will and withdraw from it. The concept of error works best in a technical rational system in which "right" is clearly defined on technical rather than moral grounds. Error is a much harder idea to apply to patients taking medication where several conflicting concepts of "good" might be at work. It would not be acceptable to label a patient's actions as an error if they were exercising an informed judgement such as stopping treatment. If we are to apply human error theory to noncompliance, we will need to amend its terminology.

It is interesting that the dominant explanatory theory of human error comes from psychology, and that psychology is probably the dominant explanatory discipline in noncompliance research. It seems likely that there could be some fruitful merging within the discipline. For example, a patient's condition changes and their response to a medicine emerges, particularly at the start of treatment when all is new. This results in the patient going through a reflexive process of reassessing their beliefs, and may result in them altering their medicine taking behaviour. While this could be categorised as a knowledge based mistake, it hardly seems to do justice to such a complex evolving process. An alternative approach is offered by some psychological models used in studying non-compliance-for example, Leventhal's self-regulatory theory. ${ }^{16}$ Work is needed to show whether these groups of theories can, or should, be effectively spliced together.

\section{CONCLUSIONS}

Non-compliance is a substantial, expensive, intractable problem in health care which can lead to misery and harm. I have argued that the theories of the causes of human error in organisations could provide a powerful explanatory model of non-compliance. If my argument is supported by empirical research, then it would lead to different approaches to reducing non-compliance in practice, to conducting research into its causes, and to the development of theory. Under this new paradigm, all deviations from directions would be taken as informative causes of non-compliance, and there would be the need to understand the relationship between minor errors and harm. Errors and non-compliance are supported by substantial literatures in the field of psychology - it seems likely that there could be some joint development of theory in this discipline. There is also a need to develop the terminology. The combined issues of the patient being the instigator of the process, the actor in the process, the recipient of the consequences of the process, and an autonomous individual (arguably, given their lack of information) would all lead to the need to reconsider the terms we use and their implicit values.

Human error theory has made significant advances over the last decade. If we can re-configure it to non-compliance and adapt it to remove terminology that may be a barrier to its adoption, then this promises to be a significant advance in our understanding and solution of non-compliance. To answer the question posed in the title of this paper, we should not consider non-compliance as a medical error as the terminology is inappropriate. However, non-compliance has a substantial amount to learn from medical error theory, and a merging of the approaches could significantly benefit patients.

\section{REFERENCES}

1 Meichenbaum D, Turk DC. Facilitating treatment adherence: a practitioner's handbook. New York: Plenum Press, 1987.

2 Sackett DL, Snow JC. The magnitude of compliance and non-compliance. In: Haynes RB, Taylor WD, Sackett DL, eds. Compliance in health care. Baltimore, London: The John Hopkins University Press, 1979: 11-22

3 Smith $M$. The cost of non-compliance and the capacity of improved compliance to reduce health care expenditures. In: Improving medication compliance. Proceedings of a Symposium held in Washington DC, November 1984. Reston, Virginia: National Pharmaceutical Council, 1985: 35-44.

4 Department of Health and Human Services. Prescription drug products: patient pack insert requirements. Fed Register 1980; 45 60754-817.

5 Lewis A. Non-compliance: a \$100bn problem. Remington Report 1997:5:14-5

6 American Society of Hospital Pharmacists. ASHP guidelines on preventing medication errors in hospitals. Am J Hosp Pharm 1993;50:305-14.

7 Haynes RB. Introduction. In: Haynes RB, Taylor WD, Sackett DL, eds. Compliance in health care. Baltimore, London: The John Hopkins University Press, 1979

8 Gordis L. Methodologic issues in the measurement of patient compliance. In: Sackett DL, Haynes RB, eds. Compliance with therapeutic regimens. Baltimore, London: The John Hopkins University Press, 1976: 51-66.

9 Stimson GV. Obeying doctor's orders: a view from the other side. Soc Sci Med 1974;8:97-104.

10 Royal Pharmaceutical Society of Great Britian/Merck Sharpe and Dohme. From compliance to concordance. Achieving shared goals in medicine taking. 1997

11 Haynes RB. Strategies for improving compliance: a methodological analysis and review. In: Sackett DL, Haynes RB, eds. Compliance with therapeutic regimens. Baltimore, London: The John Hopkins University Press, 1976: 51-66.

12 Horne R, Weinman J. Predicting treatment adherence: an overview of theoretical models. In: Myers LB, Midence K, eds. Adherence to treatment in medical conditions. Amsterdam: Harwood Academic Publishers, 1998: 25-50.

13 Haynes RB, Montague P, Oliver T, et al. Interventions for helpinmg patients to follow prescriptions for medication (Cochrane Review). In: The Cochrane Library. Issue 4. Oxford: Update Publications, 2001 (also available at http://www.update-software.com).

14 Reason JT. Human error. Cambridge: Cambridge University Press, 1990.

15 Reason JT. Understanding adverse events: the human factor. In: Vincen $\mathrm{C}$, ed. Clinical risk management. Enhancing patient safety. London: BM Books, 2001: 9-30

16 Horne R. Representations of medication and treatment: advances in theory and measurement. In: Petrie KJ, Weinman JA, eds. Perceptions of health and illness. Current research and applications. Amsterdam: Harwood Academic Publishers, 1997: 161

17 Barry CA, Stevenson FA, Britten N, et al. Giving voice to the lifeworld. More humane, more effective medical care? A qualitative study of doctor-patient communication in general practice. Soc Sci Med 2001;53:487-505.

18 Department of Health. Pharmacy in the future: implementing the NHS plan. London: Department of Health, 2000.

19 Duggan C, Feldman R, Hough J, et al. Reducing adverse prescribing discrepancies following hospital discharge. Int J Pharmacy Practice 1998;6:77-82

20 Health and Safety Executive. The costs of accidents at work. HS(G)96. London: Health and Safety Executive, 1997. 